\title{
Protective effects of flavonol isoquercitrin, against 6-hydroxy dopamine (6-OHDA) - induced toxicity in PC12 cells
}

Kasthuri Bai Magalingam ${ }^{1}$, Ammu Radhakrishnan ${ }^{1}$ and Nagaraja Haleagrahara ${ }^{2 *}$

\begin{abstract}
Background: Free radicals-induced neurodegeneration is one of the many causes of Parkinson's disease (PD). This study investigated the neuroprotective effects of flavonol isoquercitrin against toxicity induced by 6-hydroxydopamine (6-OHDA) in rat pheochromocytoma (PC12) cells.

Methods: PC12 cells were pretreated with different concentrations of isoquercitrin for 4, 8 and 12 hours and incubated with 6-OHDA for 24 hours to induce oxidative cell damage.

Results: A significant cytoprotective activity was observed in isoquercitrin pre-treated cells in a dose-dependent manner. There was a significant increase $(P<0.01)$ in the antioxidant enzymes namely superoxide dismutase, catalase, glutathione peroxidase, and glutathione in isoquercitrin pretreated cells compared to cells incubated with 6-OHDA alone. Isoquercitrin significantly reduced $(P<0.01)$ lipid peroxidation in 6-OHDA treated cells. These results suggested that isoquercitrin protects PC 12 cells against 6-OHDA-induced oxidative stress.
\end{abstract}

Conclusions: The present study suggests the protective role of isoquercitrin on 6-hydroxydopamine-induced toxicity by virtue of its antioxidant potential. Isoquercitrin could be a potential therapeutic agent against neurodegeneration in Parkinson's disease.

Keywords: Antioxidant Flavonoids 6-hydroxydopamine Parkinson's disease Oxidative stress

\section{Background}

Parkinson's disease (PD) is a neurodegenerative disease involving the degeneration of dopaminergic neurons in the striatum. The selective loss of dopaminergic neurons in the substantia nigra is the primary neuropathology in PD [1-3]. One of the many causes of PD is the accumulation of free radicals and oxidative stress which leads to the selective neuronal loss [4-6]. Though great advances have been made in the development of novel drugs to treat this disease, the appropriate pharmacological agent for PD is still elusive [3,7]. Alternative therapy in PD is aimed to effectively prevent the progression of neurodegeneration process hence improving the clinical presentation of $\mathrm{PD}$ including tremor, bradykinesia, rigor and hypokinesia [8]. Free radical scavengers such as antioxidant agents may be

\footnotetext{
* Correspondence: haleagrahara.nagaraja@jcu.edu.au

${ }^{2}$ Discipline of Physiology and Pharmacology, School of Veterinary and Biomedical Sciences, Faculty of Medicine, Health and Molecular Sciences, James Cook University, Townsville 4811, Australia

Full list of author information is available at the end of the article
}

helpful in prolonging the survival of dopaminergic neurons. Flavonoids are naturally occurring polyphenol compounds widely distributed throughout the plant kingdom $[9,10]$. They exhibit a variety of biological activities, such as anti-oxidation, anti-inflammation, anti-bacteria, and anti-allergy [11-14]. Many of these flavonoids act as neuroprotective agents in many neurological disorders $[4,15]$.

Isoquercitrin, also known as quercetin 3-glucoside, is a glucose-bound derivative of quercetin, and is reported to have anti-inflammatory and antioxidant activities $[5,6]$. Isoquercitrin is widely found in mangoes, rheum nobile, apples, onions and in many other fruits and vegetables $[16,17]$. It is considered to be a "bio-quercetin" without the potential adverse effect of quercetin [18]. Although several studies have demonstrated the antioxidant property of isoquercitrin $[5,6,16,17]$, but the neuroprotective potential of isoquercitrin is not well explored. Hence, the objectives of our study are to establish the neuroprotective role of isoquercitrin as well as to elucidate the antioxidant

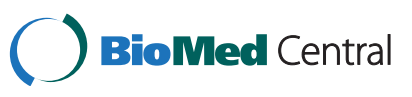


mechanisms of isoquercitrin in 6-OHDA-induced neurotoxicity in PC12 neuronal cells. PC12 cells are commonly used in the investigation of neurotherapeutics study for Parkinson's disease. These cells are known to secrete dopamine neurotransmitter and contain high amounts of dopamine transporters. The cell line is derived from the rat pheochromocytoma and is frequently used as an in vitro model to study neuronal toxicities of drugs on central dopaminergic neurons $[19,20]$.

\section{Results}

\section{Dose response of 6-OHDA toxicity}

The effect of different concentrations of 6-OHDA was assessed on 10,000 cells/well to determine the concentration of 6-OHDA which results in 50\% of cell inhibition $\left(\mathrm{IC}_{50}\right.$ value). The results showed a significant decline of cell viability following 24 hours of incubation of PC12 cells with an increasing concentration of 6-OHDA $(0-200 \mu \mathrm{M})$. The 6-OHDA concentration which resulted in 50\% PC12 cell inhibition was $100 \mu \mathrm{M}(50.33 \pm 1.72)$ compared to the negative control group. The mean percentage of cell viability of each group was compared with the mean percentage of untreated control and reported as mean \pm SEM (Figure 1).

\section{Evidence of isoquercitrin in protecting 6-OHDA- induced oxidative stress}

Isoquercitrin pre-treatment showed a significant increase in cell viability compared to cells treated with 6-OHDA alone. The $100 \mu \mathrm{M}$ of 6 -OHDA alone resulted in cell viability of $50.80 \pm 1.66$. The cell viability was the highest at $10 \mu \mathrm{M}$ of isoquercitrin at all the three durations of time. However, the optimum reading was recorded at $10 \mu \mathrm{M}$ of isoquercitrin at 8 hours of pretreatment $(73.2 \pm 1.01)$ with $p<0.001$. The cell viability was also high as the cells exposed for longer durations with isoquercitrin which is at 12 hours (Figure 2). These findings suggest that isoquercitrin could activate the native antioxidant mechanisms in the PC12 cells during the pretreatment phase, which was able to protect these cells from undergoing neurodegeneration process.

\section{Glutathione}

The glutathione level was significantly higher in the untreated sample and lower in samples incubated with 6 -OHDA alone $(\mathrm{p}<0.001)$. All samples incubated with isoquercitrin demonstrated higher amount of glutathione concentrations $(\mathrm{p}<0.001)$. The glutathione concentration was indirectly proportional to the isoquercitrin concentrations. Isoquercitrin pretreatment at $10 \mu \mathrm{M}$, showed the highest glutathione concentration in all pretreatment durations (4, 8 and 12 hours) with $\mathrm{p}<0.05$ relative to 6 OHDA treated group (Figure 3). However, the level of total glutathione was very much decreased at $100 \mu \mathrm{M}$ of isoquercitrin at 8 and 12 hour of pretreatment compared to the 4 hours of pretreatment.

\section{Glutathione peroxidase}

The GPx enzyme level was significantly higher in isoquercitrin pretreated samples compared to the sample incubated with 6 -OHDA alone $(\mathrm{p}<0.001)$. The enzyme activity increased in a dose dependent manner and the highest activity was found at $100 \mu \mathrm{M}$ of isoquercitrin pre-treated at 8 and 12 hours of incubation period. ( $p<0.05$ and $p<0.01$ ). However, isoquercitrin did not stimulate the GPx activity at concentrations lower than $100 \mu \mathrm{M}$ at all pretreatment periods as shown in Figure 4.

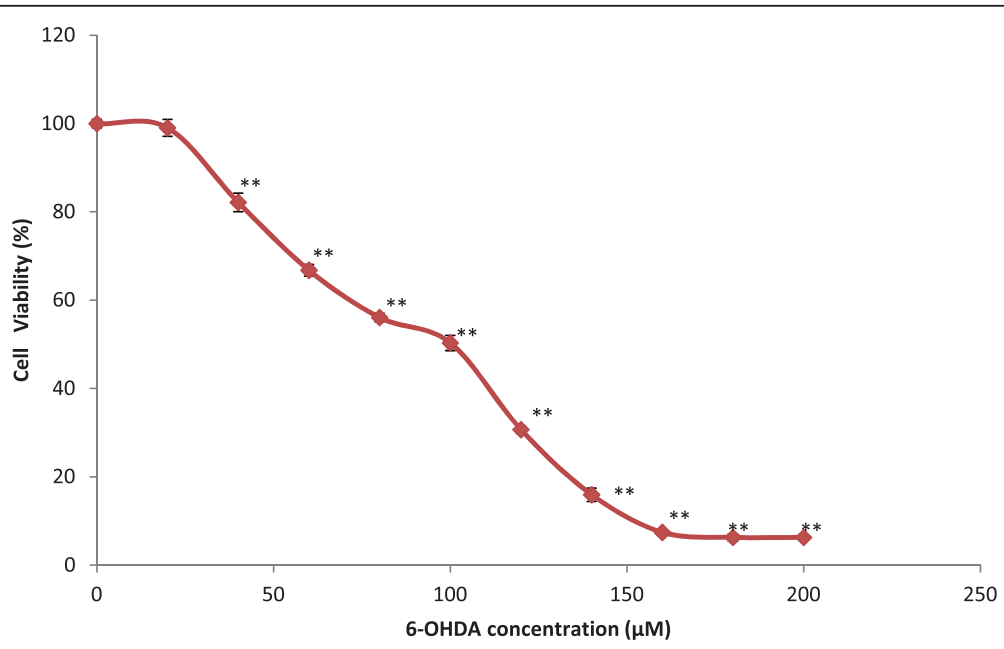

Figure 1 The dose-response of different concentrations of 6-hydroxydopamine (6-OHDA) (0-200 $\mu \mathrm{M})$ used to induce neurodegeneration in $1 \times 10^{5}$ cells/well. Pheochromocytoma (PC 12) cells for the duration of $24 \mathrm{~h}$. The viability of PC12 cells was determined using the MTT reduction assay. Values are the percentages of viable cells, with the viability of untreated control cells taken as $100 \%$. Data are mean and S.E. values from three independent experiments $(n=4) .{ }^{* *} p<0.01$, relative to untreated cells. 


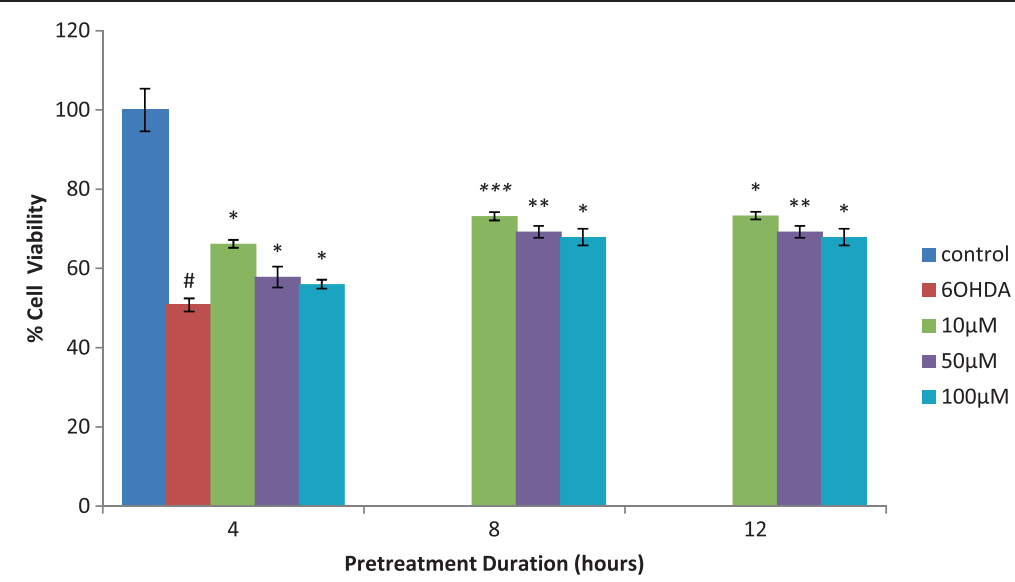

Figure 2 Assessment of cell viability after pretreatment with isoquercitrin. The isoquercitrin pre - treatment showed a significant increase in cell viability compared to cells treated with 6-OHDA alone. The cell viability was the highest at $10 \mu \mathrm{M}$ of isoquercitrin at all the three durations of time. Cell viability increased as the cells exposed for $12 \mathrm{hrs}$. with isoquercitrin. Values are the percentages of viable cells, with the viability of untreated control cells (control) taken as $100 \%$. Data are mean and S.E. values from three independent experiments $(n=3)$. \#p $<0.05$, relative to control cells. ${ }^{*} p<0.05,{ }^{* *} p<0.01$ and ${ }^{* * *} p<0.001$, relative to cells treated only with $6-\mathrm{OHDA}$.

\section{Superoxide dismutase (SOD)}

The superoxide dismutase level was significantly increased $(\mathrm{p}<0.01)$ in all isoquercitrin pretreated PC12 cells. The SOD activity did not change to a greater level as the duration of exposure with isoquercitrin was increased. However, the assay showed statistically significant elevation of SOD activity with the presence of isoquercitrin in a dosedependent manner $(\mathrm{p}<0.01)$ (Figure 5).

\section{Catalase}

Isoquercitrin showed a significant increase $(\mathrm{p}<0.01)$ in catalase activity in all pretreated cells in a dose dependent pattern. Isoquercitrin pretreated PC12 cells showed a marked increase in catalase activity in a dose-dependent manner as shown in Figure 6. The highest catalase activity was observed at $100 \mu \mathrm{M}$ of isoquercitrin at 4,8 and 12 hours of pretreatments. Therefore, we can suggest that catalase enzymes was greatly suppressed during oxidative stress (6-OHDA alone) and markedly expressed in the presence of isoquercitrin (Figure 6).

\section{Lipid peroxidation}

A significant reduction of malondialdehyde level was observed in cells treated with isoquercitrin. PC12 cells treated

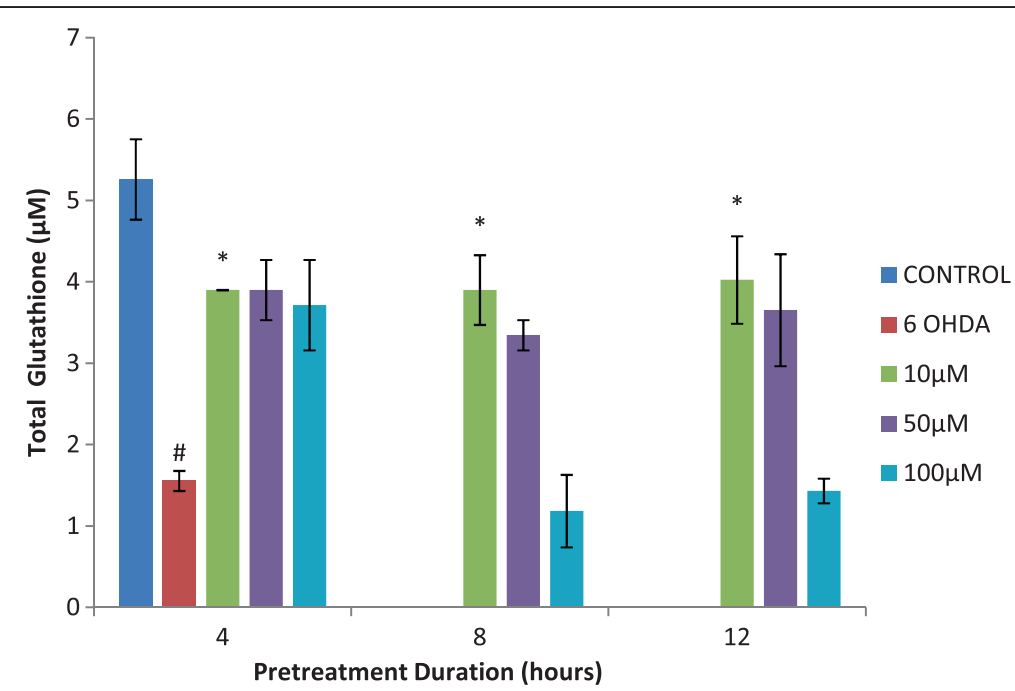

Figure 3 Effect of isoquercitrin on glutathione level. There are significantly higher glutathione in the control sample and significantly lower in samples incubated with 6-OHDA. The isoquercitrin pretreatment increased the glutathione level in 6-OHDA induced PC12 cells. Data are mean and S.E. values from three independent experiments $(n=3)$. $\# p<0.05$, relative to control cells. ${ }^{*} p<0.05$, relative to cells treated only with 6-OHDA 


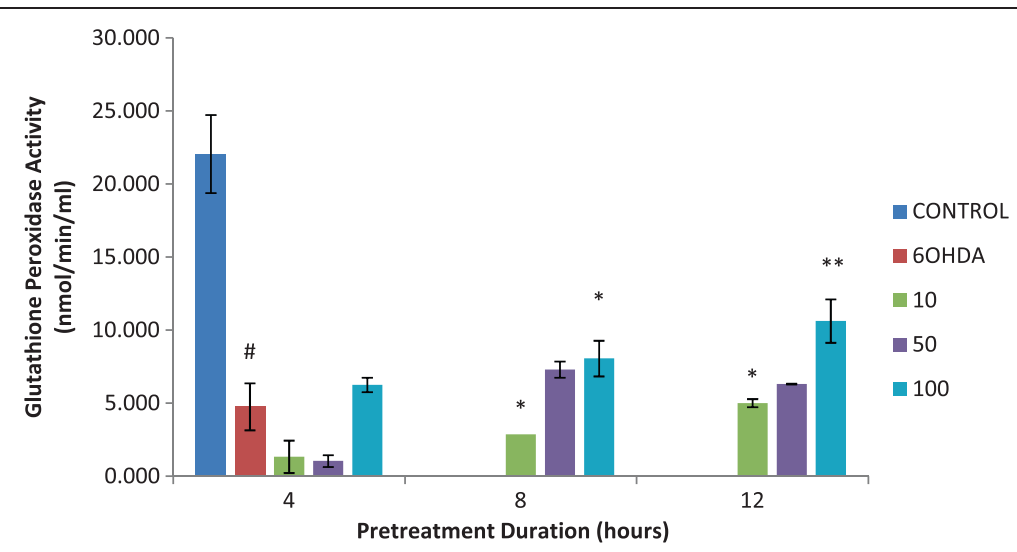

Figure 4 Effect of isoquercitrin on glutathione peroxidase activity. The enzyme level was significantly higher in isoquercitrin pretreated samples. Glutathione peroxidase activity increased in a dose dependent manner. Data are mean and S.E. values from three independent experiments $(n=3)$. $\# p<0.05$, relative to control cells. ${ }^{*} p<0.05$ and ${ }^{* *} p<0.01$, relative to cells treated only with $6-\mathrm{OHDA}$.

with 6-OHDA alone showed a remarkably high level of malondialdehyde which is an indication of lipid peroxidation or oxidative stress. Isoquercitrin significantly reduced the lipid peroxidation in a dose-dependent manner. The highest concentration of isoquercitrin showed the greatest potential in suppressing lipid peroxidation in PC12 cells as the MDA level was the least in that group $(100 \mu \mathrm{M})$. All the test groups displayed significant results with $\mathrm{p}<0.01$ relative to 6-OHDA treated cells alone (Figure 7).

\section{Discussion}

Many lines of evidence have proven the oxidative stress due to imbalance in the free radical generation and endogenous antioxidant defense system may lead to the selective neuronal loss in Parkinson's disease [21-23]. Hence, flavonoid polyphenols, particularly isoquercitrin can be an ideal candidate as neuroprotective agent to cease or delay the degeneration of dopaminergic cells $[24,25]$. In our study, the neuroprotective role of isoquercitrin, a flavonoid glycoside was investigated using a 6-OHDA induced PC12 cellular model of Parkinson's disease. In the cell viability assay, isoquercitrin pretreatment has demonstrated a remarkable increase of cell viability at 12 hours of incubation with $10 \mu \mathrm{M}$ of isoquercitrin. The isoquercitrin pretreated prior to 6-OHDA exposure resulted in stimulation of antioxidant enzyme activity in the neuronal cells as the cells are more resilient in coping with upcoming oxidative stress [26,27]. The antioxidant enzyme defense system functions in eliminating the free radical induced cellular damage during the defense against microorganisms, toxic chemicals and other conditions of cellular stress $[20,21,28]$.

To further validate the neuroprotective role of isoquercitrin, the antioxidant enzyme status of isoquercitrin

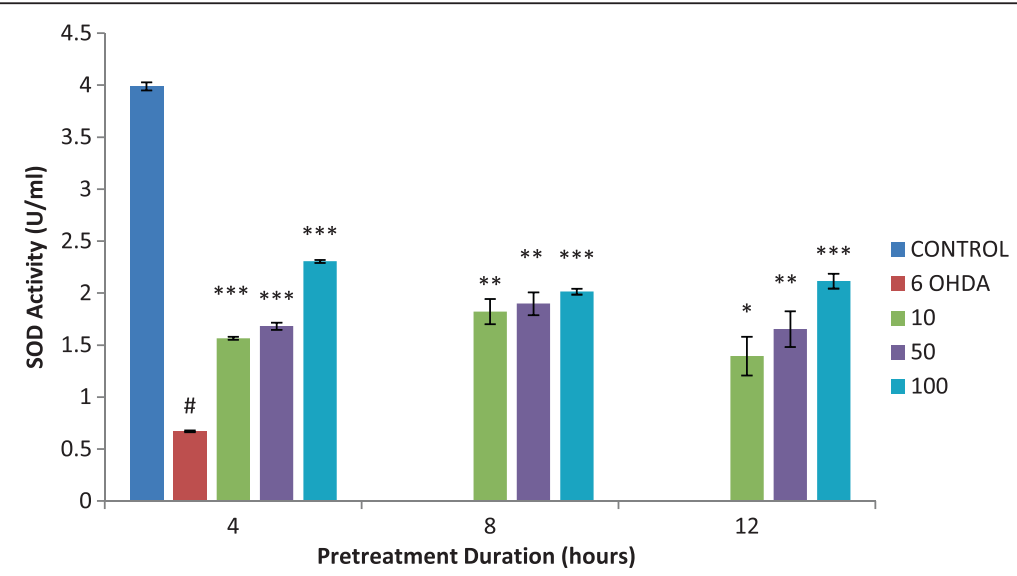

Figure 5 Effect of isoquercitrin on superoxide dismutase activity. The SOD significantly increased in all isoquercitrin pretreated cells. There was a significant elevation of SOD with the presence of isoquercitrin in a dose-dependent manner. Data are mean and S.E. values from three independent experiments $(n=3)$. $\# p<0.001$, relative to control cells. ${ }^{*} p<0.05$, ${ }^{* *} p<0.01$ and ${ }^{* *} p<0.001$, relative to cells treated only with 6-OHDA. 


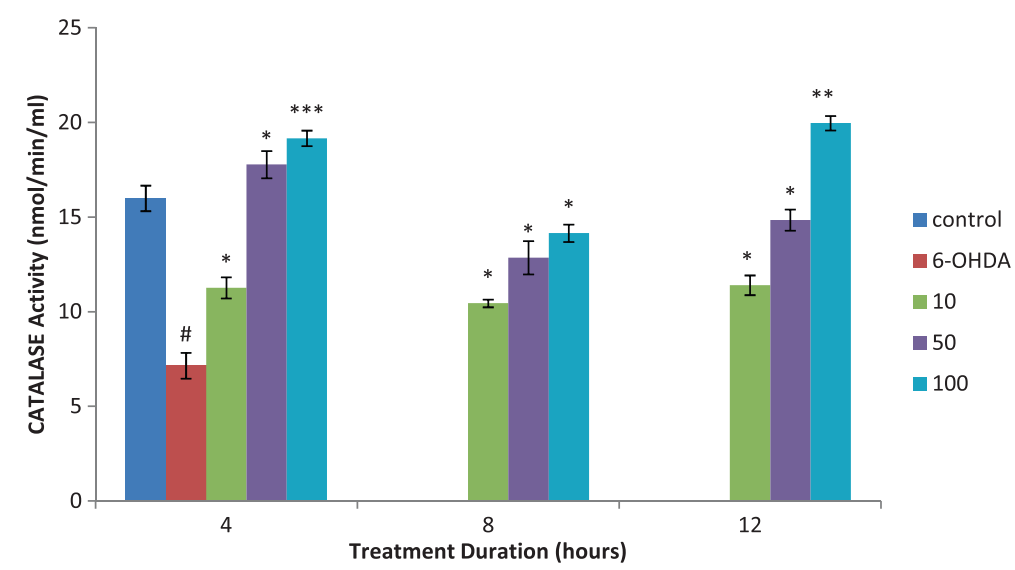

Figure 6 Effect of isoquercitrin on catalase activity. Isoquercitrin showed a significant increase in the catalase activity in all pretreated cells in a dose dependent pattern. The greater activity of catalase was seen after 4 and 12 hours. Data are mean and S.E. values from three independent experiments $(\mathrm{n}=3)$. $\# p<0.01$, relative to control cells. ${ }^{*} p<0.05,{ }^{* *} p<0.01$ and ${ }^{* * *} p<0.001$, relative to cells treated only with $6-\mathrm{OHDA}$.

pretreated PC12 cells was assessed to confirm the antioxidant capability of this flavonol. The antioxidant enzymes which were studied are superoxide dismutase (SOD), catalase (CAT), glutathione and glutathione peroxidase (GPx). SOD occur in significantly higher amounts in the brain and these enzymes, which are of three types $(\mathrm{Cu}-\mathrm{Zn} \mathrm{SOD}$, $\mathrm{Mn}-\mathrm{SOD}$ and EC-SOD) readily catalyze the dismutation of the superoxide anion to oxygen molecules and hydrogen peroxide, a less toxic molecule [21,29]. There was a significant increase in SOD in all the isoquercitrin pretreated cells in a dose dependent manner. This increase in SOD activity proved that there was a direct activation of SOD by isoquercitrin to catalyze the superoxide anions produced by 6-OHDA. Catalase, a tetrameric structure with four indistinguishable tetrahedrally arranged residues with a single ferri-protoporphyrin subunit is ubiquitously found in the liver, kidney and erythrocytes [30]. Catalase accounts for detoxifying $\mathrm{H}_{2} \mathrm{O}_{2}$ molecules, whereby it is converted to oxygen and water molecule and this reaction through a reaction known as the catalytic reaction [31]. The CAT activity in isoquercitrin pretreated 6-OHDA induced PC12 cells showed a statistically significant effect in all the treatment groups. The CAT activity in the 6-OHDA treated group was significantly reduced compared to control group. The increased CAT activity could be due to two mechanisms, (i) increased in hydrogen peroxide molecules by SOD triggered the CAT enzyme, (ii) isoquercitrin caused direct activation of CAT enzyme which catalyzed the toxic hydrogen peroxide to water and oxygen molecules $[29,32]$.

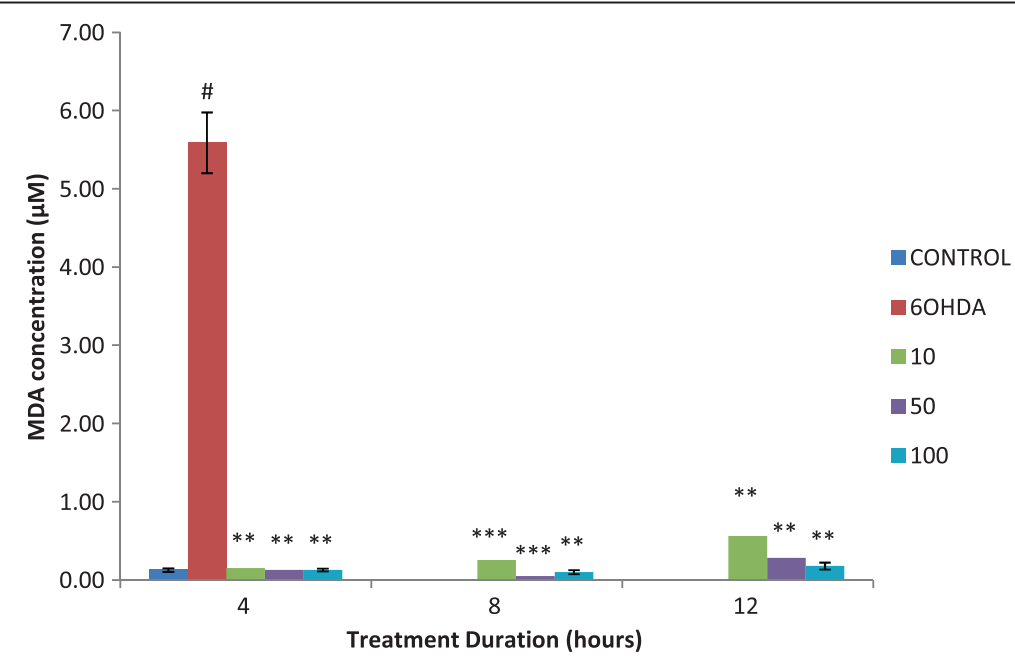

Figure 7 Effect of isoquercitrin on lipid peroxidation. Isoquercitrin significantly reduced the lipid peroxidation in a dose-dependent manner. The highest concentration of isoquercitrin showed the greatest potential in suppressing lipid peroxidation in PC12 cells. Data are mean and S.E. values from three independent experiments $(n=3)$. $\# p<0.01$, relative to control cells. ${ }^{* *} p<0.01$ and ${ }^{* * *} p<0.001$, relative to cells treated only with 6-OHDA. 
Glutathione peroxidase, which is largely found in the cytoplasm and mitochondria of eukaryotic cells is a vital antioxidant enzyme that catalyzes the reduction of hydroperoxides [33]. In this study, the GPx/Glutathione activity was also increased by antioxidant treatment. Isoquercitrin probably interacted with GPx and glutathione to enhance their antioxidant activity in PC12 cells $[21,34]$. Malondialdehyde (MDA) is a naturally occurring product of lipid peroxidation that will react with the thiobarbituric acid (TBA) and form MDA-TBA adducts [35]. Malondialdehyde produced due to lipid peroxidation accumulated in the cells and cause cell damage. MDA increased in 6OHDA treated cells and isoquercitrin effectively reduced the levels of MDA in the pre-treated cells [27-29,32].

Free radicals generated by neurotoxin, 6-hydroxydopamine caused neuronal cell loss via DNA defects, lipid peroxidation and cytoskeletal disorganization [34]. Moreover, studies also demonstrated that 6-OHDA induced neuronal loss was due to inhibition of the mitochondria respiratory chain complexes I and IV, oxidative phosphorylation uncoupling, mitochondrial membrane potential collapse [4,22,35,36]. In this study, we have proved that 6-hydoxydopamine induced oxidative stress and cell death by decreasing the scavenging enzymes (SOD, catalase and GPx) in PC 12 cells. Isoquercitrin pretreatment caused a significant elevation in these scavenging enzyme levels and attenuate oxidative damage to the cells $[22,37,38]$. Isoquercitrin helped to maintain the levels of these antioxidant enzymes and suppress lipid peroxidation as well as protect the neuronal cell from undergoing cell death.

\section{Conclusions}

The results of this study confirmed that neurotoxin 6hydroxy dopamine causes suppression of antioxidant enzyme levels in PC 12 cells, which could be a reason for the progressive neuronal death. Isoquercitrin's role in protecting against 6-OHDA induced oxidative stress, suggests that this flavonol may serve as a potential neuroprotective agent against the underlying pathology associated with neurodegenerative diseases like Parkinson's disease.

\section{Methods \\ Materials}

PC12 cells were purchased from ATCC (\#CRL-1721.1 PC12 ADH, RattusNorvegicus). 6-hydroxydopamine, isoquercitrin, poly-L-Lysine, MTT (3-(4,5-Dimethylthiazol2-yl)-2,5-diphenyltetrazolium bromide),3,4 - dihydroxyL-phenylalanine (Levodopa) and dimethyl sulphoxide (DMSO) were purchased from Sigma-Aldrich (USA). Dulbecco's modified eagle's medium (DMEM), pen-strep, horse serum, and fetal bovine serum was purchased from GIBCO Inc. (USA). Kits for glutathione peroxidase, superoxide dismutase, catalase, thiobarbiturate assay, and glutathione were purchased from the Cayman Chemical Company (USA).

\section{Cell culture}

PC12 cells were grown in a humidified incubator with $5 \% \mathrm{CO}_{2}$ at a temperature of $37^{\circ} \mathrm{C}$ in DMEM medium supplemented with $5 \%$ horse serum and $5 \%$ fetal bovine serum and pen-step $(100 \mathrm{U} / \mathrm{ml})$. The cells were cultured in poly-L-lysine coated T-75 culture flasks. The cells used in the experiments were taken between passage 2 and 8 as cells tend to get clumpy and difficult to isolate after passage 10 . When the cells were $80 \%$ confluent, they were dislodged from the flask using a cell scraper. The dispersed cells were grown on poly-L-lysine coated 96-well microplate at a density of $1 \times 10^{5} \mathrm{cells} / \mathrm{ml}$ and allowed overnight incubation to facilitate cell adhesion to the substrate. The cells were treated for 4,8 and 12 hours in the presence of isoquercitrin at three different concentrations, $10 \mu \mathrm{M}, 50 \mu \mathrm{M}$ and $100 \mu \mathrm{M}$. Subsequently, the pre-treated cells were induced using 6-OHDA for 24 hours and assayed for its antioxidant activities. Control cells were cultured in complete DMEM alone and positive control cells were treated only with 6-OHDA.

\section{Determination of cell viability}

The cytotoxicity effect of isoquercitrin pretreatment on 6OHDA induced PC12 was determined using MTT (3-(4, 5-Dimethylthiazol-2-yl)-2,5-diphenyltetrazolium bromide assay. MTT, a yellow tetrazole, is reduced to insoluble purple formazan in the mitochondria of viable cells and appears purple. The insoluble purple formazan was dissolved using a solubilizing solvent and the colored solution was measured at $570 \mathrm{~nm}$ using a microplate-reader. Data on cell viability were expressed as percentage of the surviving control cell in the study.

\section{Biochemical parameters}

Glutathione assay kit utilizes an optimized enzymatic recycling method that uses glutathione reductase for quantification of GSH. Rate of production of yellow colored 5Thio-2-nitrobenzoic acid (TNB) is directly proportional to the recycling reaction and concentration of glutathione in the sample. Lipid peroxides, derived from polyunsaturated fatty acids, are unstable and decompose to form a complex series of compounds which include reactive carbonyl compounds, such as MDA. MDA-TBA adducts formed by the reaction of MDA and TBA under high temperature and acidic conditions is measured colorimetrically at $550 \mathrm{~nm}$. Super oxide dismutase assay kit utilizes a tetrazolium salt for the detection of superoxide radicals $(\mathrm{O} 2-)$ generated by xanthine oxidase and hypoxanthine. One unit of SOD is defined as the amount of an enzyme necessary to exhibit $50 \%$ dismutation of superoxide radical. Superoxide dismutase levels were determined from a standard curve and 
expressed as $\mathrm{U} / \mathrm{ml}$ of protein. Glutathione peroxidase assay kit measures GPx activity indirectly by a coupled reaction with glutathione reductase. Oxidized glutathione, produced upon reduction of an organic hydroperoxide by $\mathrm{GPx}$, is recycled to its reduced state by glutathione reductase and NADPH. The oxidation of NADPH to NADP + is accompanied by a decrease in absorbance at $340 \mathrm{~nm}$. Glutathione peroxidase levels were determined from a standard curve and expressed as $\mathrm{nmol} / \mathrm{min} / \mathrm{ml}$ of protein. The Cayman chemical catalase kit utilizes the peroxidatic function of catalase for determination of enzyme activity. The assay is based on the reaction of catalase with methanol in the presence of an optimal concentration of $\mathrm{H} 2 \mathrm{O} 2$. The formaldehyde produced is measured spectrophotometrically with 4-amino-3-hydrazino-5-mercapto-1,2,4triazole as the chromogen.

\section{Statistical analysis}

Data were expressed as mean \pm SEM. The results were analyzed using analysis of variance (ANOVA) using SPSS Inc. software (SPSS Statistics, V20.0.0). Differences between treatment groups were determined using the Bonferonni post-hoc test. A value of $\mathrm{P}<0.05$ was considered statistically significant.

\section{Competing interests}

The authors declare that they have no competing interests.

\section{Authors' contributions}

KBM performed all experiments and wrote the manuscript. AR and HN designed the overall study and prepared the final manuscript. All authors read and approved the final manuscript.

\section{Author details}

'Department of Pathology, Faculty of Medicine, International Medical University, Kuala Lumpur, Malaysia. ${ }^{2}$ Discipline of Physiology and Pharmacology, School of Veterinary and Biomedical Sciences, Faculty of Medicine, Health and Molecular Sciences, James Cook University, Townsville 4811, Australia.

Received: 17 August 2013 Accepted: 16 January 2014 Published: 21 January 2014

\section{References}

1. Covy JP, Giasson BI: Alpha-Synuclein, leucine-rich repeat kinase-2, and manganese in the pathogenesis of Parkinson disease. Neurotoxicology 2011, 2011(32):622-629.

2. Garcia Ruiz PJ, Catalan MJ, Fernandez Carril JM: Initial motor symptoms of Parkinson disease. Neurologist 2011, 17:18-S20.

3. Ybot-Gorrin I, Vivancos-Matellano F, Chacón-Peña JR, Alonso-Navarro H, Jiménez-Jiménez FJ: Assessment of Parkinson disease: what do we need to show neuroprotection? Neurologist 2011, 17:S21-S29.

4. Glinka Y, Gassen M, Youdim MB: Mechanism of 6-hydroxydopamine neurotoxicity. J Neural Transm 1997, 50:55-66.

5. Rogerio AP, Kanashiro A, Fontanari C, da Silva EV, Lucisano-Valim YM, Soares $E G$, Faccioli LH: Anti-inflammatory activity of quercetin and isoquercitrin in experimental murine allergic asthma. Inflamm Res 2007, 56:402-408.

6. Jung $\mathrm{SH}, \mathrm{Kim} \mathrm{BJ}$, Lee $\mathrm{EH}$, Osborne NN: Isoquercitrin is the most effective antioxidant in the plant Thujaorientalis and able to counteract oxidativeinduced damage to a transformed cell line (RGC-5 cells). Neurochem Int 2010, 57:713-721.

7. Chen S, Le W: Neuroprotective therapy in Parkinson disease. Am J Ther 2006, 13:450-457.
8. Shimohama S, Sawada H, Kitamura Y, Taniguchi T: Disease model: Parkinson's disease. Trends Mol Med 2003, 9:360-365.

9. Hertog MGL, Hollman PCH, van de Putte B: Content of potentially anticarcinogenic flavonoids of 28 vegetables and 9 fruits commonly consumed in the Netherlands. J Agric Food Chem 1992, 40:2379-2383.

10. Mu X, He GY, Cheng Y, Li X, Xu B, Du G: Baicalein exerts neuroprotective effects in 6-hydroxydopamine-induced experimental parkinsonism in vivo and in vitro. Pharmacol Biochem Behav 2009, 92:642-648.

11. Pietta PG: Flavonoids as antioxidants. J Nat Prod 2000, 63:1035-1042.

12. García-Lafuente A, Guillamón E, Villares A, Rostagno MA, Martínez JA: Flavonoids as anti-inflammatory agents: implications in cancer and cardiovascular disease. Inflamm Res 2009, 58:537-552.

13. Alcaráz LE, Blanco SE, Puig ON, Tomás F, Ferretti FH: Antibacterial activity of flavonoids against methicillin-resistant Staphylococcus aureus strains. J Theor Biol 2000, 205:231-240.

14. Cheong H, Ryu SY, Oak MH, Cheon SH, Yoo GS, Kim KM: Studies of structure activity relationship of flavonoids for the anti-allergic actions. Arch Pharm Res 1998, 21:478-480

15. Agati G, Azzarello E, Pollastri S, Tattini M: Flavonoids as antioxidants in plants: location and functional significance. Plant Sci 2012, 196:67-76.

16. Cornard JP, Boudet AC, Merlin JC: Theoretical investigation of the molecular structure of the isoquercitrin molecule. J Mol Struct 1999, 508:37-49.

17. Lee S, Park HS, Notsu Y, Ban HS, Kim YP, Ishihara K, Hirasawa N, Jung SH, Lee YS, Lim SS, Park EH, Shin KH, Seyama T, Hong J, Ohuchi K: Effects of hyperin, isoquercitrin and quercetin on lipopolysaccharide induced nitrite production in rat peritoneal macrophages. Phytother Res 2008 22:1552-1556

18. Gasparotto A Jr, Gasparotto FM, Boffo MA, Lourenço EL, Stefanello ME, Salvador MJ, da Silva-Santos JE, Marques MC, Kassuya CA: Diuretic and potassium-sparing effect of isoquercitrin-An active flavonoid of Tropaeolummajus L. J Ethnopharmacol 2011, 134:210-215.

19. Jenner P: Oxidative damage in neurodegenerative disease. Lancet 1994 344:796-798.

20. Walkinshaw G, Waters CM: Neurotoxin-induced cell death in neuronal PC12 cells is mediated by induction of apoptosis. Neuroscience 1994, 63:975-987.

21. Datta K, Sinha S, Chattopadhyay P: Reactive oxygen species in health and disease. Natl Med J India 2000, 13(6):304-310.

22. Dauer W, Przedborski S: Parkinson's disease: mechanisms and models. Neuron 2003, 39:889-909.

23. Gilgun-Sherki Y, Melamed E, Offen D: Oxidative stress induced neurodegenerative diseases: the need for antioxidants that penetrate the blood brain barrier. Neuropharmacol 2001, 40:959-975.

24. Vauzour D, Vafeiadou K, Rodriguez-Mateos A, Rendeiro C, Spencer JP: The neuroprotective potential of flavonoids: a multiplicity of effects. Genes Nutr 2008, 3:115-126.

25. Chanh PH, Ifansyah N, Chahine R, Mounayar-Chalfoun A, Gleye J, Moulis C: Comparative effects of total flavonoids extracted from Ribesnigrum leaves, rutin and isoquercitrin on biosynthesis and release of prostaglandins in the ex vivo rabbit heart. Prostaglandins Leukot Med 1986, 22:295-300.

26. Lobo V, Patil A, Phatak A, Chandra N: Free radicals, antioxidants and functional foods: Impact on human health. Pharma Rev 2010, 4:118-126.

27. Wei Z, Bai OJ, Richardson S, Mousseau DD, Li XM: Olanzapine protects PC12 cells from oxidative stress induced by hydrogen peroxide. J Neurosci Res 2003, 73:364-368.

28. Allen RG, Tresini M: Oxidative stress and gene regulation. Free Radic Biol Med 2000, 28:463-499.

29. Reiter RJ: Oxidative damage in the central nervous system: protection by melatonin. Prog Neurobiol 1998, 56:359-384

30. Yoshimoto M, Sakamoto H, Yoshimoto N, Kuboi R, Nakao K: Stabilization of quaternary structure and activity of bovine liver catalase through encapsulation in liposomes. Enzyme Microb Technol 2007, 41(6):849-858.

31. Singh $R$, Wiseman B, Deemagarn $T$, Jha V, Switala J, Loewen PC: Comparative study of catalase-peroxidases (KatGs). Arch Biochem Biophys 2008, 471:207-214

32. Nehlig A: The neuroprotective effects of cocoa flavanol and its influence on cognitive performance. Br J Clin Pharmacol 2013, 75:716-727.

33. José MM, Cristina PM, Ignacio ND: Antioxidant enzymes and human diseases. Clin Biochem 1999, 32:595-603.

34. Blum D, Torch S, Lambeng N, Nissou M, Alim-Louis B, Sadoul R, Verna J: Molecular pathways involved in the neurotoxicity of 6-OHDA, dopamine 
and MPTP: contribution to the apoptotic theory in Parkinson's disease. Prog Neurobiol 2001, 65:135-172.

35. Niedernhofer L, Daniels JS, Rouzer CA, Greene RE, Marnett LJ:

Malondialdehyde, a product of lipid peroxidation, is mutagenic in human cells. J Biol Chem 2003, 278:31426-31433.

36. Qu CP, Xu ZR, Liu GJ, Liu C, Li Y, Wei ZG, Liu GF: Differential expression of copper-zinc superoxide dismutase gene of Polygonumsibiricum leaves, stems and underground stems, subjected to high-salt stress. Int J Mol Sci 2010, 11:5234-5245.

37. Haleagrahara N, Siew CJ, Kumar P: Effect of quercetin and desferrioxamine on 6-hydroxydopamine (6-OHDA) induced neurotoxicity in striatum of rats. J Toxicol Sci 2013, 38:25-33.

38. Yamada K, Umegaki H, Iguchi A, Kameyama T, Nabeshima T: Possible involvement of catalase in the protective effects of interleukin- 6 against 6-hydroxydopamine toxicity in PC12 cells. Brain Res Bull 1997, 46:573-577.

doi:10.1186/1756-0500-7-49

Cite this article as: Magalingam et al:: Protective effects of flavonol isoquercitrin, against 6-hydroxy dopamine (6-OHDA) - induced toxicity in PC12 cells. BMC Research Notes 2014 7:49.

\section{Submit your next manuscript to BioMed Central and take full advantage of:}

- Convenient online submission

- Thorough peer review

- No space constraints or color figure charges

- Immediate publication on acceptance

- Inclusion in PubMed, CAS, Scopus and Google Scholar

- Research which is freely available for redistribution 